Sādhanā Vol. 38, Part 5, October 2013, pp. 897-924. (C) Indian Academy of Sciences

\title{
Electronics in nuclear power programme of India-An overview
}

\author{
G P SRIVASTAVA
}

Electronics and Instrumentation Group, Bhabha Atomic Research Centre, Mumbai 400 085, India

e-mail: gyan25@yahoo.com

\begin{abstract}
This paper presents an overview of state-of-the art developments in electronics for nuclear power programme of India. Indigenous activities in instrumentation and control (I\&C) in the areas of detector development, nuclear instrumentation, monitoring and control electronics and special sensors paved the way to self-reliance in nuclear industry. Notable among the recent I\&C systems developed for 540 MWe reactors are Liquid Zone Control System (LZCS), flux mapping system and advance reactor regulating system. In a nuclear plant, apart from ensuring functional requirements, design of electronics needs to meet high level of reliability, safety and security standards. Therefore, a lot of importance is attached to activities such as design review, testing, operation, maintenance and qualifications of I\&C systems. Induction of computer based $\mathrm{I} \& \mathrm{C}$ systems mandated a rigorous verification process commensurate with the safety class of the system as specified in Atomic Energy Regulatory Board (AERB) safety guides. Software reliability is assured by following strict development life cycle combined with zero-defect policy and is verified through verification and validation $(\mathrm{V} \& \mathrm{~V})$ process. Development of new techniques in data transmissions with optical fibres as transmission medium and wireless networks in control systems is being pursued. With new I\&C systems, efforts were made to utilize the same hardware and software platforms for various plant applications, i.e., for standardization. Thrust was given to use Field Programmable Gate Arrays (FPGA) and Application Specific Integrated Circuits (ASIC) in order to improve the reliability of system by reducing component count. It has become imperative to develop modern contemporary solutions like ASICs, HMCs, System on Chip (SOC) and detector mounted electronics and towards that various ASICs and HMCs have been developed in-house to meet the challenges.
\end{abstract}

Keywords. Nuclear power programme; nuclear instrumentation; instrumentation and control; computer based systems; verification and validation.

\section{Introduction}

Electronics and instrumentation (E\&I) is an important and integral component in the entire nuclear fuel cycle program of India ranging from extraction, fabrication, power production, 
reprocessing and waste management. It has a very crucial role in radiation protection measures also. The instrumentation and control system architecture, together with plant operating personnel, makes the 'central nervous system' of a nuclear power plant (NPP) or the facility. Historically right from APSARA days, most of the I\&C activities in Indian nuclear program have been supported by research and developments undertaken at BARC in the areas of electronics, instrumentation, communications, high performance computing and information security. The production has been well-supported by Electronics Corporation of India Limited.

Each I\&C system in a nuclear power plant, through its elements (e.g., equipment, sensors, transmitters, actuators, etc.), senses basic physical parameters, monitors performance, processes information, and makes automatic adjustments to plant operations as necessary. It also responds with appropriate action to process failures and off-normal events, thus ensuring plant and personnel safety, simultaneously meeting the goals of efficient power production. I\&C systems need to meet not only the functional, performance and interface requirements but also the enhanced reliability, safety and security standards. Therefore, a lot of importance is given to activities involving the design, design review, testing, operation, maintenance and qualifications of I\&C systems.

I\&C systems provide protection, control, supervision and monitoring in NPPs. Some of these functions are for safety critical, while others are safety related, and those which may have indirect influence on safety and availability. NPP systems and equipments are classified (or categorized) depending on their relationship to plant safety. In general, a graded classification approach is used whereby the more direct relationship of a system to a safety function, the higher is its classification. This allows the systematic application of appropriate design and engineering techniques and, just as importantly, helps to avoid over-design. Extensive developmental activities have been taken up for the ongoing research and power reactor programs; for Light Water Reactors (LWR), Prototype Fast Breeder Reactor (PFBR), Advanced Heavy Water Reactor (AHWR) and the critical facilities.

Indigenous development program of highly specialized and customized sensors for nuclear reactor applications are integral part of I\&C systems. These sensors are designed to meet the specific application requirements, high pressure, high temperature, nuclear radiation endurance, and provide adequate vibration resistance. The radiation detector development programme has produced variety of detectors to meet the $I \& C$ requirement of the indigenous nuclear power program. The requirements of monitoring the reactivity status of the reactor core from a specified subcritical state to beyond full power of the plant influences mechanical design of the reactor core internals to locate appropriate detectors. Variety of neutron detectors used in nuclear instrumentation for the indigenous nuclear reactors viz. Dhruva, Kamini, Fast Breeder Test Reactor (FBTR), PFBR, LWR and PHWR have been developed in-house and qualified.

In NPPs, the practice of design and implementation of computer based systems (CBS) for carrying out functions important to safety has matured over last several years and it is possible to demonstrate their fitness-for-purpose. Computer-based systems are being employed for control functions, monitoring of equipment, processes, radiation and important parameters, test and monitoring of various safety systems and operator information and support functions. Notable amongst is the development of new C \& I systems for the 540 MWe reactors Tarapur Atomic Power Station (TAPS) $3 \&$ 4. For improved reliability and maintainability the reactor protection system has been fully computerized for the first time. In order to have more number of inputs and output functions, it was necessary to have a new multi-nodal system architecture for the Reactor Regulating System (RRS) featuring use of digital communication for 
the first time in a safety related system. A new type of reactivity control device called Liquid Zone Control Compartment has also been developed for the 540 MWe reactors. The Flux Mapping System (FMS) for 540 Mwe was designed for the detection of xenon-induced oscillations and to control them. Computerized Fuel Handling Control System has been deployed in 14 operating PHWRs in India. Radiation monitoring system has been designed to limit external and internal radiation exposure received by persons outside the site boundary and by plant personnel.

Computer-based systems for $\mathrm{I} \& \mathrm{C}$ are mandated to undergo a rigorous verification process commensurate with the safety class of the system as specified in AERB safety guides D-10, D-20. Effectiveness of defence-in-depth is enhanced with channel and system independence. Performance requirement in terms of target reliability and single failure criteria are satisfied with minimal spurious operation. Requirements of Fail-safe design, testability, maintainability, qualification of the systems specified in the standards for general I\&C systems are applicable to CBS with some additional requirements detailed in D-25. The design requirements are assured by building-in testability in every module. Software reliability is assured by following strict development life cycle combined with zero-defect policy and is verified through V\&V process. Along with D-25, IEC-60880 is followed for complete software development process and for $\mathrm{V} \& \mathrm{~V}$ process of the safety systems. Less-stringent IEC-62138 is followed for class IB and IC systems.

NPP I\&C systems are generally isolated from external communication systems. Nonetheless, particularly the computers used in safety and safety-related systems must be very well-protected from possible intrusions. Systems are protected physically against unauthorised access and virus attack. Apart from that, in CBS, the software and data are protected allowing only authorised access. This is done by having multiple levels of access control like password and hardware keys. Remote access to the software is not allowed from outside the specified technical environment. The systems are not connected to any general purpose networks. BARC has a well-defined program on I\&C security. Security plans have been developed which controls the development and deployment of critical software/hardware for NPPs. The software verification and validation activities specifically scrutinize the products from security related aspects in all phases of its development. Security is built into the systems, security tests are done. Any security threats, security vulnerabilities are studied and protected against.

With new I\&C systems efforts were made to utilize the same hardware and software platforms for various plant applications, i.e., standardization. The program on standardized hardware modules for use in future reactor and other applications is being pursued continuously. These modules are designed based on present day technology and components for the life of the plant. Thrust was given to use Field Programmable Gate Arrays (FPGA) and Application Specific Integrated Circuits (ASIC) in order to improve the reliability of system by reducing component count. This would also help against obsolescence and constraints associated with import of electronic components. Qualified Trombay Programmable Logic Controller-32 (TPLC-32) platform has been designed to provide configurability options with inbuilt safety features. For AHWR as a next generation nuclear power plant a modern control room has been conceived for operation and monitoring of the plant.

Plant control system architectures have been evolved in the last few decades. While the speed of network (latency and the redundancy) governed the media and protocol, this helped in reduction of number of cables and elimination of radio frequency interference problems at the plant level. Optical fibres as the medium and new techniques like Wavelength divison multiplexing (WDM) in data transmissions have been developed and are being proven. Work on wireless networks in control systems is being pursued. 
Development of silicon detector has been carried out which find wide range of applications in several fields such as high energy particle physics, nuclear physics and astrophysics research, imaging for security applications, medical imaging, high resolution $\alpha$ and charged particle spectroscopy, detection of low activity radiation, personal dosimeters for $\beta, \gamma$ and neutrons, medical dosimetry, low energy X-ray spectroscopy, etc. The rapid growth of semiconductor technology it has become imperative to develop modern contemporary solutions like ASICs, HMCs, System on Chip (SOC) and detector mounted electronics. In this direction various ASICs and HMCs have been developed to meet the challenges and upgrade the instrumentation.

\section{Detectors and sensors}

\subsection{Neutron detectors}

Nuclear instrumentation uses various types of detectors to measure neutron flux during all states of nuclear reactor operation. Among the various types of detectors thus used are boron lined proportional counters, fission counters, $\mathrm{BF}_{3}$ counters, uncompensated and gamma compensated boron lined ionization chambers and self-powered neutron detectors. The type of detector for a specific application is chosen based on the range of neutron flux to be measured, the ambient temperature, the ambient gamma background at the detector location and service life. The mechanical design of the detector, the material of construction, choice of insulators, the type of gas-fill, etc., are made to ensure long-term stable operation of these detectors. Several types of boron-10 lined neutron detectors and fission counters are developed and being used in research reactors such as Dhruva and KAMINI and compact light water reactors. Based on BARC technology, ECIL produced detectors are deployed in Indian PHWRs.

Since neutrons are uncharged particles, their detection is carried out by causing them to interact with materials that generate energetic charged particle following neutron interaction. The materials so chosen include Boron enriched in B-10, He-3 and U-235 or U-233. B-10 is introduced into the sensitive volume of the detector either as $\mathrm{BF}_{3}$ gas or as a thin deposit of elemental boron on the detector electrodes. U-235 or U-233 is introduced as thin deposits of their oxides on the electrodes. Neutrons interacting with any of the above materials generate energetic charged particles, which ionizes the gas in the sensitive volume of the detector. These ionized particles are collected either as a single pulse or as a DC current and the signal is proportional to the neutron flux incident on the detector. B-10 lined and He-3 detectors are used in the proportional region in the pulse mode operation to enhance the signal strength. Over the years the detector technology for reactor use has matured to produce robust detectors. The main specifications of some of the detectors are given in table 1.

\subsection{Nuclear instrumentation for reactors}

Nuclear instrumentation, consisting of radiation detectors and signal processing channels is deployed in a nuclear reactor to measure the fission power produced in the reactor core and the rate of change of fission power at any instant. The nuclear instrumentation is typically deployed to perform the reactor safety, control as well as the monitoring functions of the reactor core. The reactor active state for core fission rate measurement shall start from effective core multiplication 


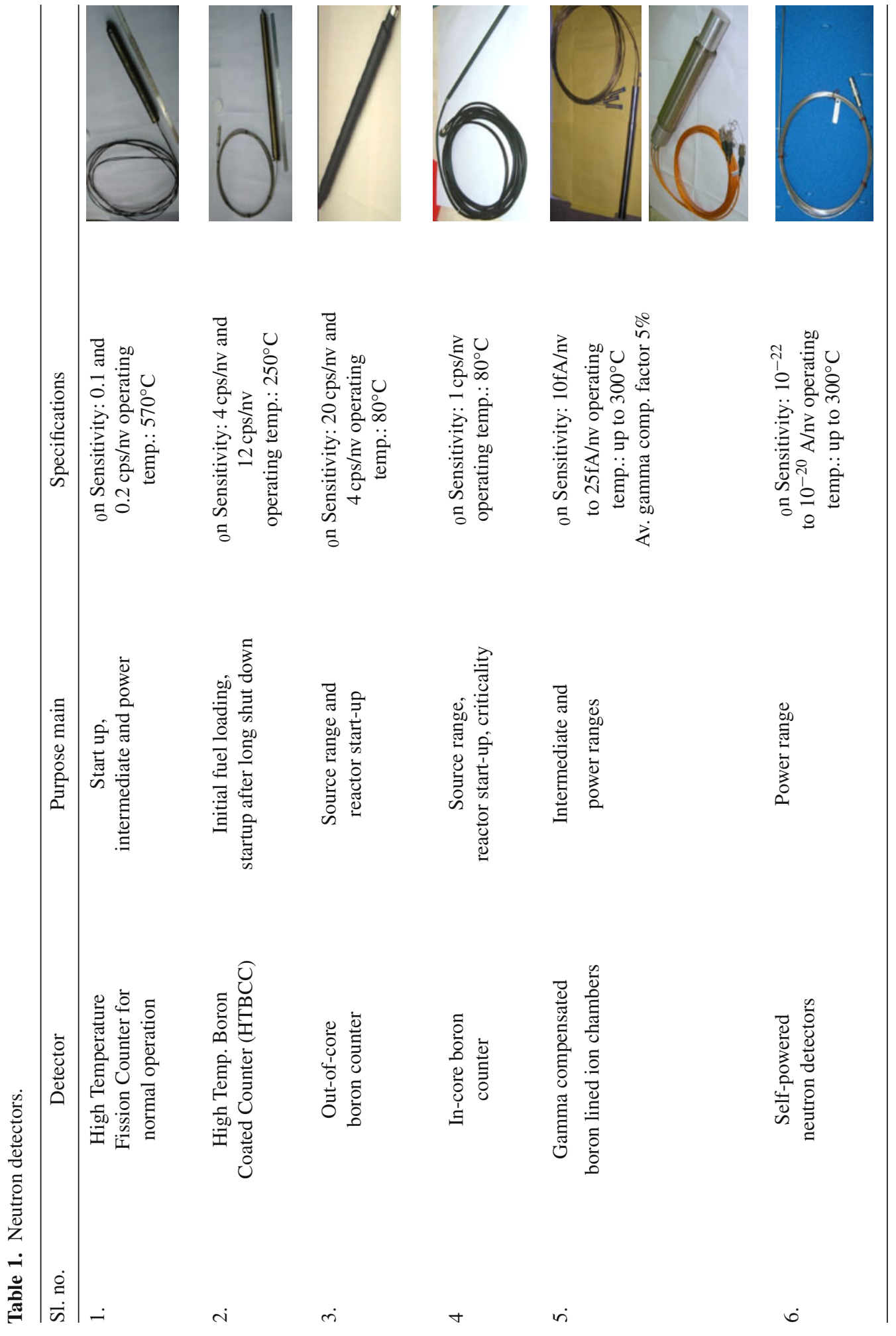


factor $\left(\mathrm{K}_{e f f}\right)$ of 0.95 and continue to measure beyond full power typically $150 \%$ Full power (FP). Thereby, nuclear instrumentation is deployed to measure the core fission rate in all the states of the reactor viz. shutdown, startup, intermediate and power range including the fuel loading/refuelling in the core. The measurement range of the nuclear instrumentation is well over twelve decades.

The credible signal from nuclear instrumentation implies that the fission rate value generated by the instrumentation is statistically accurate within the desired band and is generated within the required response time. At low fission rates in the reactor core, the individual neutrons falling on the detector are counted over a time and rate of pulses produced per unit time represents the fission rate at that instant. Since individual neutron interaction with the detector is random in time, the accumulated pulse counts over a time determine the statistical accuracy of pulse count rate. The minimum count rate and the acceptable counting time determine the value of credible signal in 'pulse' mode of operation. The detectors such as ${ }^{10} \mathrm{~B}$ Lined proportional counter, $\mathrm{BF}_{3}$ counters and Fission Counter operate in pulse mode. In pulse mode, the instrumentation is designed to process the detector pulses faithfully and generate count rate of the detector pulses to represent the fission rate. In other case, the detectors are operated in DC modes when the neutron flux incident on the detector is significantly higher and the average DC signal current is the measure of neutron flux. The average DC current produced should be at least 10 times higher than the detector leakage/noise currents. The detectors such as ${ }^{10} \mathrm{~B}$ coated Ionization chambers-gamma uncompensated or compensated, Fission chambers and self-powered neutron detectors (SPNDs) operate in DC mode. There exists a part in the total measurement range where at some higher counting rates the pulse counting results in high pulse counting loss $(>10 \%)$ due to pulse pile up and at the same time the average DC currents are too low to be measured accurately. This portion of the measurement range is termed as intermediate range. The intermediate range employs the technique of 'Mean Square Voltage' (MSV) to discriminate the noise currents and to measure neutron signal accurately till the DC signal becomes stronger at higher power. This technique of measurement in intermediate range is also popularly known as 'Campbell mode' (Dubridge 1967). The required signals derived using the measured neutron flux are reactor power, reactor period and the reactivity.

The detectors operating in pulse mode produce small charge signal typically of the order of $0.1-0.3 \mathrm{pC}$ for a time duration of $80-300 \mathrm{nsec}$ for every interaction with the thermal neutron. The detector amplifier in reactor application is located generally at a distance of 50-150 meters. Thereby special coaxial (Triax/Super-screened) cables are used for carrying the signal up to the amplifier with special considerations on shielding and separation from other plant cables to minimize the EMI pickups. The signal processing of pulse mode detectors generally employ amplifiers operating in current mode. Subsequent to amplification the pulses are discriminated against a threshold to eliminated noise and other radiation signals. The gross counting of the pulses is used to determine the count rate that represents neutron flux at the detector location. The logarithm of the count rate signal is generated to cover wide range of about six decades. The derivative of the log count rate signals is generated to derive the reactor period. The detectors operating in DC current mode typically produce currents of the order of $10^{-14} \mathrm{Amp} / \mathrm{nv}$ and use logarithmic amplifier to generate the neutron flux and subsequently the reactor period information. In Campbell range the neutron flux information is generated by calculating the variance of the signal received from the detector to achieve higher discrimination against gamma radiation. The reactivity is computed by on-line solving the inverse point kinetic equation using six delayed group of neutrons. The randomness in interaction of thermal neutrons within the detector results in the statistical fluctuations in the processed signal which are minimized by incorporating appropriate averaging time. 
BARC has been engaged in design and development of nuclear instrumentation for core neutron flux measurement of nuclear reactors starting from APSARA in 1950s, followed by Zerlina, Dhruva, Kamini, FBTR, IPHWRs and so on. Typically, Light Water Reactors (LWRs) require detectors located inside the core and also at the out of core locations. Whereas, the IPHWRs of small core (220 MWe) use only the neutron detectors located at the out of core locations for normal start-up and operation of the reactor. The IPHWRs of large core (540 MWe) require large number of in core detectors for control and safety besides the out of core detectors. All IPHWRs require different set of in core detectors for special start-up. The fast reactor (FBTR) also use out of core neutron detectors and measure the thermal equivalent flux at the detector location.

The nuclear instrumentation of PFBR employs High Temperature $\left(600^{\circ} \mathrm{C}\right)$ Fission Chambers (HTFC) for normal operation including regular reactor start-up. The reactor power operation utilizes multi-section fission chamber. There high temperature $\left(250^{\circ} \mathrm{C}\right)$ Boron $\left({ }^{10} \mathrm{~B}\right)$ coated counters to be used for initial fuel loading, first approach to criticality and startup after long shut down. These detectors in combination measure neutron flux for over ten decades from shutdown to full power in normal mode of reactor operation which consist of pulse, Mean Square Voltage' (MSV) and DC current modes of operation. The instrumentation scheme is triplicated for safety and dual for control.

The nuclear instrumentation for Indian Light Water Reactor of specific type monitors the core neutron flux and primary coolant temperature under all conditions of reactor including shutdown. The neutron flux is monitored over ten decades range using out-core detectors of two types-one fission counters and other gamma compensated ionization chambers.

The development of low noise fast circuit components for processing circuits has improved the signal to noise ratio appreciably. The development efforts are towards making neutron detectors with improved sensitivity and long reactor in-situ life. In signal processing front, there has been significant achievement in improving the signal to noise ratio of detector signal processing, however the noise immune circuits and self test features will enhance the detection of slow degradation and maintainability of the instrumentation. Besides these, some of the development areas include direct estimation of core multiplication factor at any time by noise analysis techniques and improving the counting statistics at lower end of measurement range with improved response time.

\subsection{Kalman filtering-based online instrument for indication of instantaneous reactivity in nuclear reactors}

In reactor operation or experiments, signals indicating reactor power (or neutron flux) and reactor period are used for direct information on the state of the reactor. However, the most important time dependent parameter is reactivity, and in the past few decades, many techniques have been proposed by researchers for on-line dynamic computation of reactivity. Many of the digital reactivity meters employ reactivity estimation based on inverse point kinetic equations or otherwise based on reactor period. However, these algorithms demand an accurate knowledge of the model parameters, which is difficult to provide in many cases. Also they do not have any direct provision to eliminate noise content in the data, and hence fail miserably in estimation of sub-criticality when the neutron flux level is low. Kalman Filtering algorithm, with its inherent ability to work with even highly noisy input signals and uncertain plant parameters, is found to be very promising in such situations.

The Kalman Filter is a recursive stochastic estimator algorithm, which uses a mathematical model of the process to predict the instantaneous values of all the process variables; including 
those which cannot be directly measured. It further corrects these predicted values using the available measurements from the actual process. This predictive-corrective process, with in-built noise filtering capability, repeats in each cycle of the algorithm execution. A reactivity meter based on this algorithm has been developed for providing the reactor operator with a direct indication of the core reactivity.

The reactivity meter unit is an embedded system consisting of processor with add-on data acquisition and analog output cards. It accepts as inputs the signals corresponding to reactor power from the neutronic channels, and provides indication of the estimated reactivity through a Graphical User Interface (GUI) panel as shown in figure 1 or an analog meter mounted on the control panel in Main Control Room.

During the past decade, efficacy of the meter in estimation of core reactivity under different operating conditions has been demonstrated in different types of reactors. Vast operational experience from these exercises has proved that the reactivity meter is a very handy tool in applications such as estimation of reactivity perturbations around critical conditions and indication of point of criticality during reactor start-up, shutdown margin monitoring, worth computation and calibration of reactivity devices, detection of insertion/removal of small amounts of reactivity under subcritical conditions, etc.

The use of a digital reactivity meter for real-time indication of sub0criticality is a challenging problem, essentially due to the difficulties in modelling the neutron source strength with sufficient accuracy, and also due to the high fluctuations in neutron signals measured in subcritical systems. The Kalman filter algorithm has inherent capability to work with stochastic signals and hence addresses the latter issue efficiently. Former issue is taken care of by an explicit modelling of the neutron source in terms of any known initial sub-criticality as mentioned previously. Efficacy of the meter in indication of sub-criticality has been validated in research reactors and critical facility at Trombay and in Fast Breeder Test Reactor (FBTR), Kalpakkam. Figure 2 depicts the performance of the meter in the critical facility during a transient involving lowering of regulating rod followed by dropping of safety rods. The online estimation was found to be in good agreement with the values calculated offline by conventional methods.

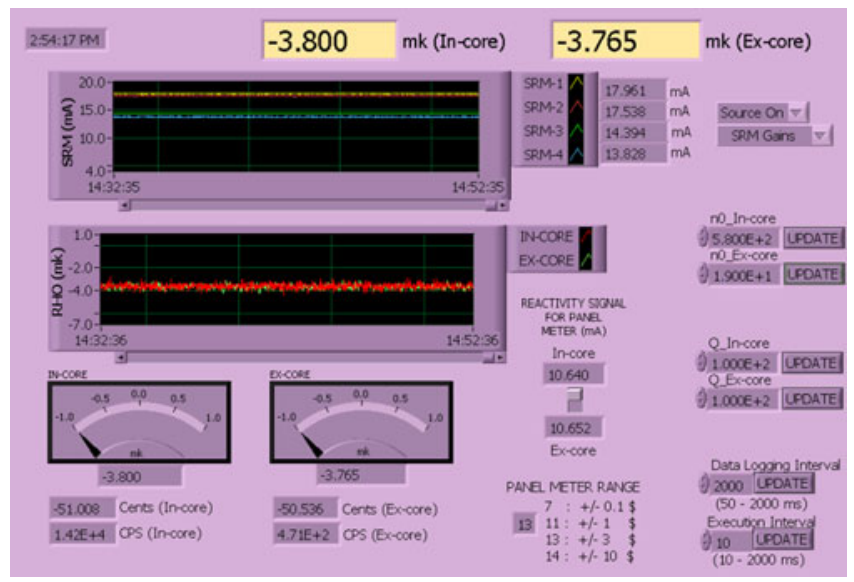

Figure 1. Screenshot of the reactivity meter GUI. 

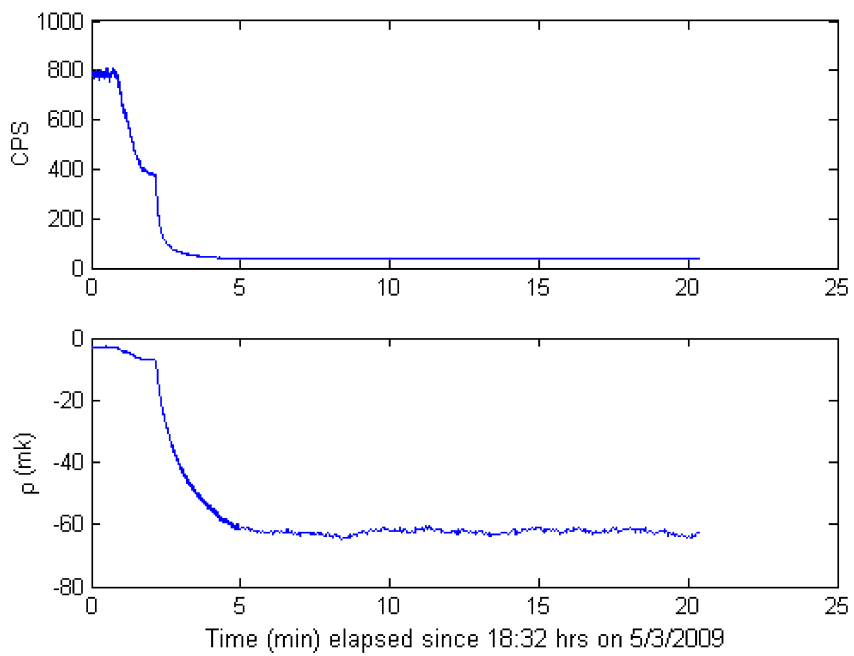

Figure 2. Sub-criticality indication in the critical facility during the lowering of regulating rod.

\subsection{Worth computation and calibration of reactivity devices}

Experiments conducted at different reactors demonstrated the capability of the meter to serve as a handy tool for direct online computation of the worth of different reactivity devices and their calibration. Outcome of one such exercise in the critical facility is depicted in figure 3 . It shows the worth estimation when two shut-off rods were withdrawn sequentially. Likewise, figure 4 depicts the response during step-by-step withdrawal and subsequent insertion of the regulating rod in the critical facility.
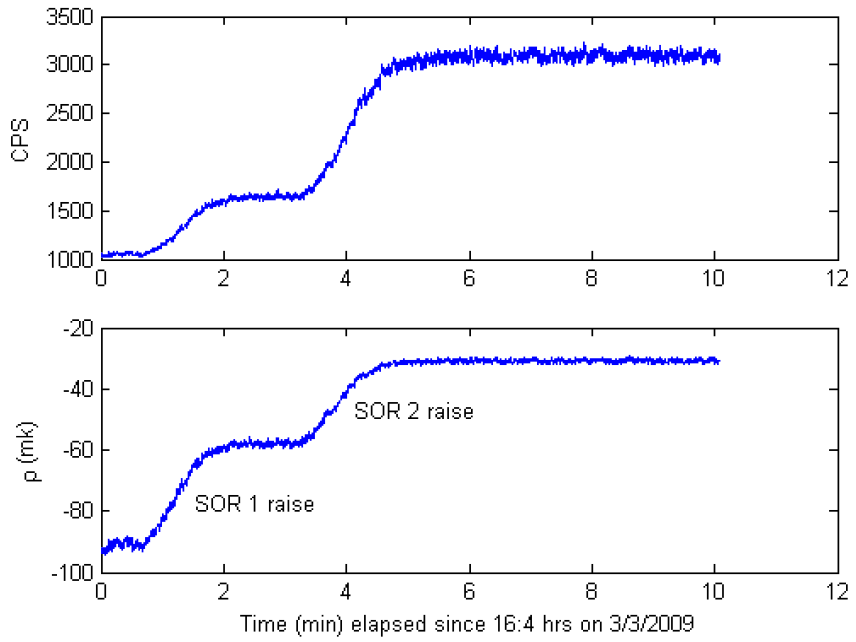

Figure 3. Online estimation of shutoff rods' worth in critical facility. 

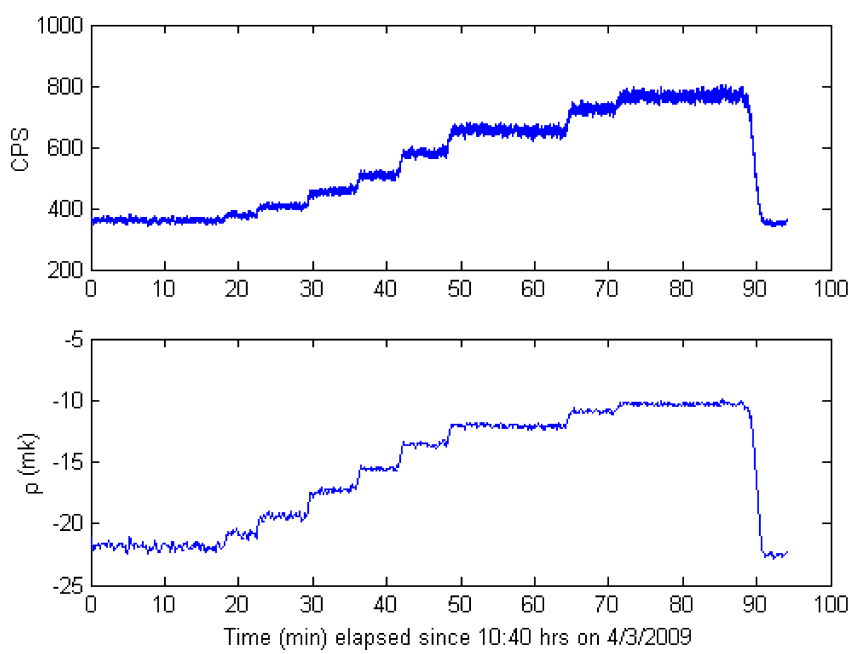

Figure 4. Indicated reactivity during regulating rod calibration exercise in critical facility.

\subsection{Process sensors}

Process sensors are used in process systems of nuclear reactor plant for monitoring, plant control, and protection of reactor and actuation of safety systems. Process sensors measure the parameters like temperature, pressure, flow rate, water level of reactor and reactor auxiliary systems, steam and feed water systems, reactor containment system and engineered safety systems.

BARC has developed special sensors for nuclear reactor applications as the customized application requirements that could not be met by commercially available sensors. Sensors of the safety systems which are mounted in the containment area are designed to withstand the normal ambient conditions and also the ambient conditions which exist during severe accident like LOCA. The sensors support remotely mounted electronics so that the electronics can be kept out of accident zone and the sensor is located inside the containment. The sensors are designed with remotely mounted electronic converter. Sensors connected to Primary Heat Transport (PHT) system of the plant are designed to achieve zero leakage, welded process connection. Remote health check facility provided in sensor electronic converters which enables sensor testability on command from the operator console. These include ultrasonic level sensors, absolute pressure sensors, flow meters and salinity sensors.

\section{Control systems for 540 MWe pressurized heavy water reactors}

The commissioning the two 540 MWe PHWR units located at Tarapur represents significant milestone as India entered, with this, into an era of electricity generation from nuclear reactors of large size. This achievement has been possible through assimilation of interdisciplinary activities, involving several contributors. Mathematical modelling, simulation and control of space-time kinetics phenomena; hardware-in-loop simulation of liquid zone control system; and real-time computation of complex control functions for generation of feedback signals for reactivity devices by employing a distributed multi-nodal architecture are some such contributions made by BARC. 
The 540 MWe PHWR is a large reactor requiring spatial control which in simple terms means control of power distribution along with the control of total power, so as to keep the xenoninduced oscillations from growing. Design of spatial control system necessitated, on one hand, the modelling of the space-time kinetics phenomena and on the other, selection of a suitable control design method for handling Multiple Input Multiple Output (MIMO) problems of large order.

The control system which is designed using a mathematical model of the system is tested for its performance by simulation, before implementation. For this, a facility comprising of a core simulator, Reactor Regulating System and reactivity control mechanisms (Adjuster rods, Control rods and Liquid Zone Control System (LZCS) was used and the different parameters in control algorithms were optimized. Among the different reactivity control mechanisms in $540 \mathrm{MWe}$ PHWR, the LZCS is the most important for fine control of reactor power and power distribution. It consists of 14 zone control compartments (ZCC) contained in 6 vertical LZCA units placed appropriately in the calandria. As LZCS was being used for the first time in our reactors, the need was felt for understanding the behaviour of the system before its installation in TAPS 3 \& 4. A replica of the LZCS of TAPP $3 \& 4$ in respect of equipment sizes, layout and elevations was set-up and commissioned in 2002 at RCnD, BARC.

The procedure for commissioning of the LZCS (bringing the water levels in ZCC and helium pressure in delay tank, storage tank, helium inlet header, and helium outlet header at desired values without causing flooding of ZCCs and excessive variations in helium outlet header pressure) was established. The procedure was successfully followed in commissioning of the system at TAPP 4. A number of process parameters and operational ranges were finalized based on experience gained with the test set-up.

With the experience that was gained on the LZCS test set-up, installation and commissioning of LZCS at TAPP 4 was very smoothly carried out and some problems that surfaced were easily understood and solved. Detailed study on the LZC System along with the core and RRS simulator outside the reactor environment has paid far beyond its cost by way of savings in expenditure through reduction of overall commissioning time at Tarapur site.

\section{Computer-based Systems (CBS) in NPP}

Introduction of computer-based systems in NPP has been achieved in phased manner. As the confidence in the deployed systems increased, more and more systems have been computerised. It is logical to introduce information systems first as computer-based systems in NPPs. PHWR has unique feature of having pressure tubes inside caldaria tubes, instead of pressure vessel as in BWRs and PWRs. Large number of such tubes exist and contains fuel and coolant passing through it, temperature of which are to be monitored. In MAPS, the first CBS channel temperature monitoring systems (CTM) were deployed. This system was monitoring and displaying the temperatures of various channels in chromatic profile format, for easy assimilation of information by operator and generates alarms on temperature crossing certain limits. It also had facility for tuning the lead compensation for each measurement channel, which was done during hotconditioning, through operator interface. Subsequently, from Kaiga onwards, advanced versions of CTMs were designed which were generating setback signal and hence are of safety-related class.

Digital recording systems (DRS), later also named as Disturbance Recording System was the other information system installed and commissioned at Kakrapar NPP and later on deployed at MAPS and NAPS. This becomes handy for analysis after an event, including trip. Event sequence recorder (ESR) was designed to record the events in the chronological order. 


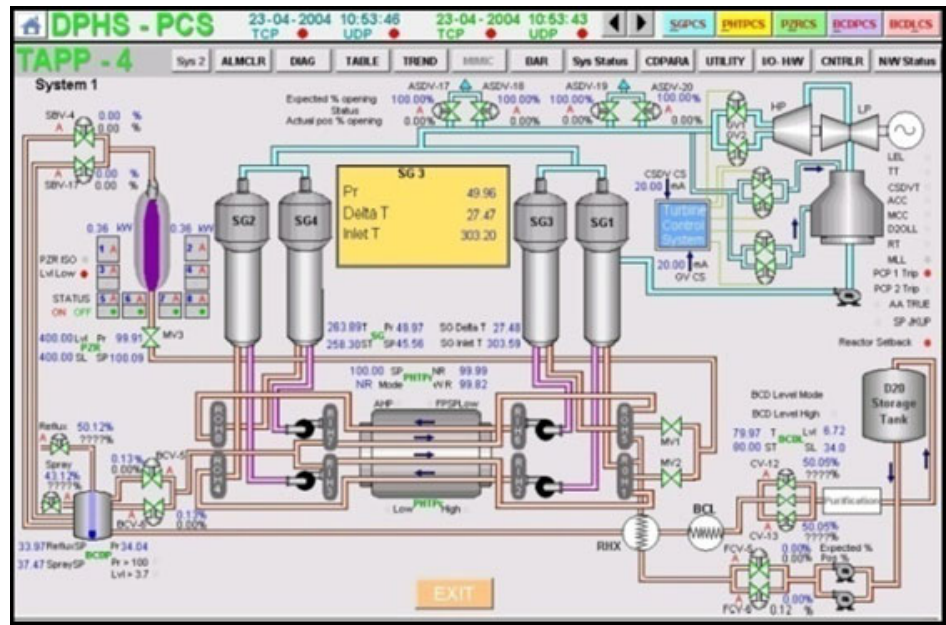

Figure 5. Mimic in OI of DPHS-PCS system.

Power measurement and automatic control of reactor power by controlling reactivity devices is done by reactor regulating system (RRS). The first system was deployed at Narora and Kakrapar. Taking technological advances into account, advanced RRS was designed and deployed at subsequent plants from Kaiga onwards, of having dual processor and hot-standby (DPHS) configuration, providing better features. 540 MWe at Tarapur is the large reactor and have large number of reactivity devices to be controlled. It also performs set-back, step back functions, provides xenon override facility, does zone control of reactivity and also does rod movement monitoring of various types of rods. This system also does withdrawal of the shut-off-rods on start-up. This advanced RRS was designed as multi-nodal system, with networking. Since this reactor is a large reactor, a flux-mapping system, also of multi-nodal configuration was designed and built; this system provides on-line compensation for zone power variations and also provides three-dimensional information to operator on neutron flux across the reactor (figures 5, 6).

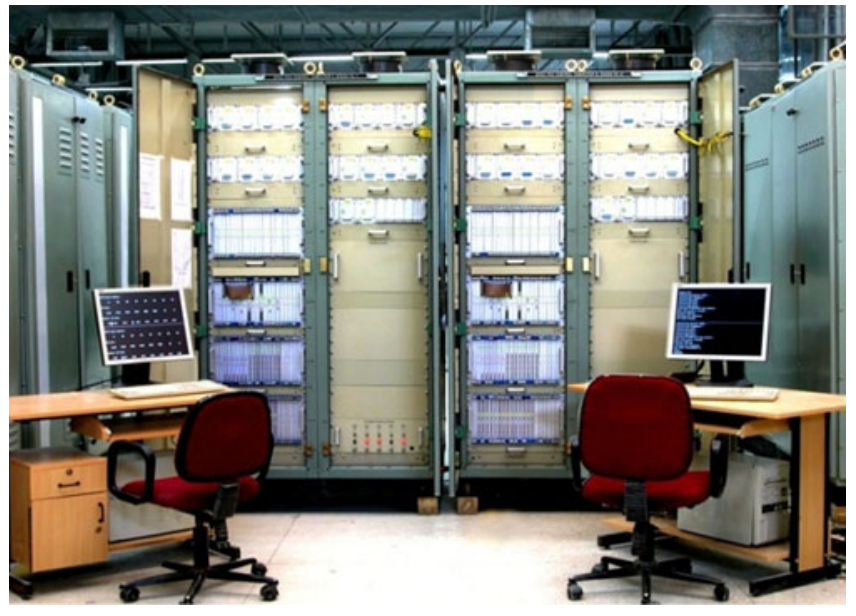

Figure 6. DPHS-PCS hardware for TAPP-4. 
Large core size of 540 MWe PHWR is loosely coupled and because of which xenon-induced power oscillations can set in the reactor. There is a need to provide special systems for detection of xenon-induced oscillations and to control them. The Flux Mapping System (FMS) (refer figure 7) for 540 MWe PHWR is first of its kind used in Indian PHWRs. FMS computes flux/power distribution profile in the reactor core as shown in figure 8 using modal synthesis method, detects xenon-induced oscillations and to control them together with Reactor Regulating System (RRS). Additionally, FMS also performs computation of burn up of $\mathrm{Co}^{59}$ isotope and the buildup of $\mathrm{Co}^{60}$ isotope in cobalt Self-Powered Neutron Detectors (SPND). To facilitate ease in monitoring and control of the reactor power distribution, the reactor is divided into 14 zones. The power in each of these 14 zones is measured using prompt responding cobalt self-powered neutron detectors (SPNDs) connected to RRS. However, the zone power estimates of RRS derived using cobalt SPNDs need correction. FMS provides accurate estimate of zone power distribution within the core based on slow responding vanadium self-powered neutron detectors.

Computer-based process control system for controlling SG pressure, primary coolant pressure, bleed condenser pressure and level was first time introduced in Kaiga and continued with improved features for other reactors. Networked, PLC-based systems are in use for various auxiliary systems, handling functionalities of safety class IB, IC, NINS (Not Important for Nuclear Safety) from Kaiga reactors onwards. The Supervisory Control and Data Acquisition (ESCADA) System at RAPS-3\&4 for the electrical systems provides operators in the Main Control Rooms and in the Switchyard Control Room have the capability to monitor and control the status of various electrical equipment in the nuclear power station.

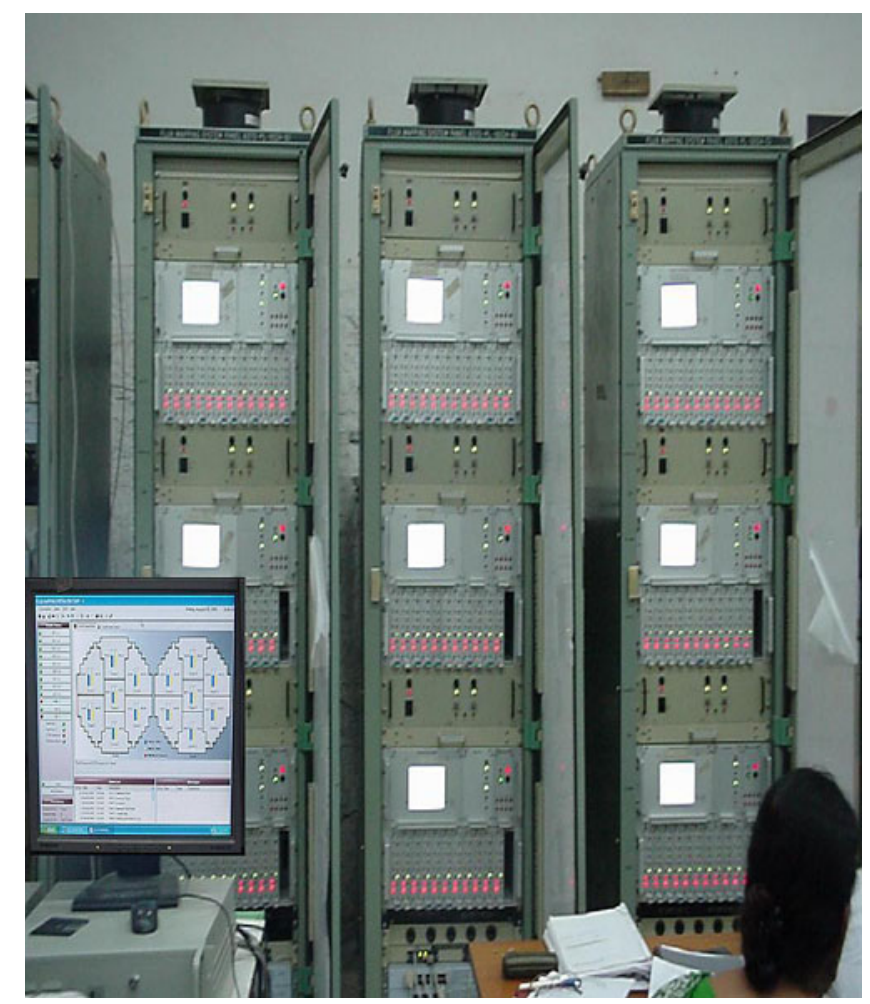

Figure 7. Flux mapping system. 


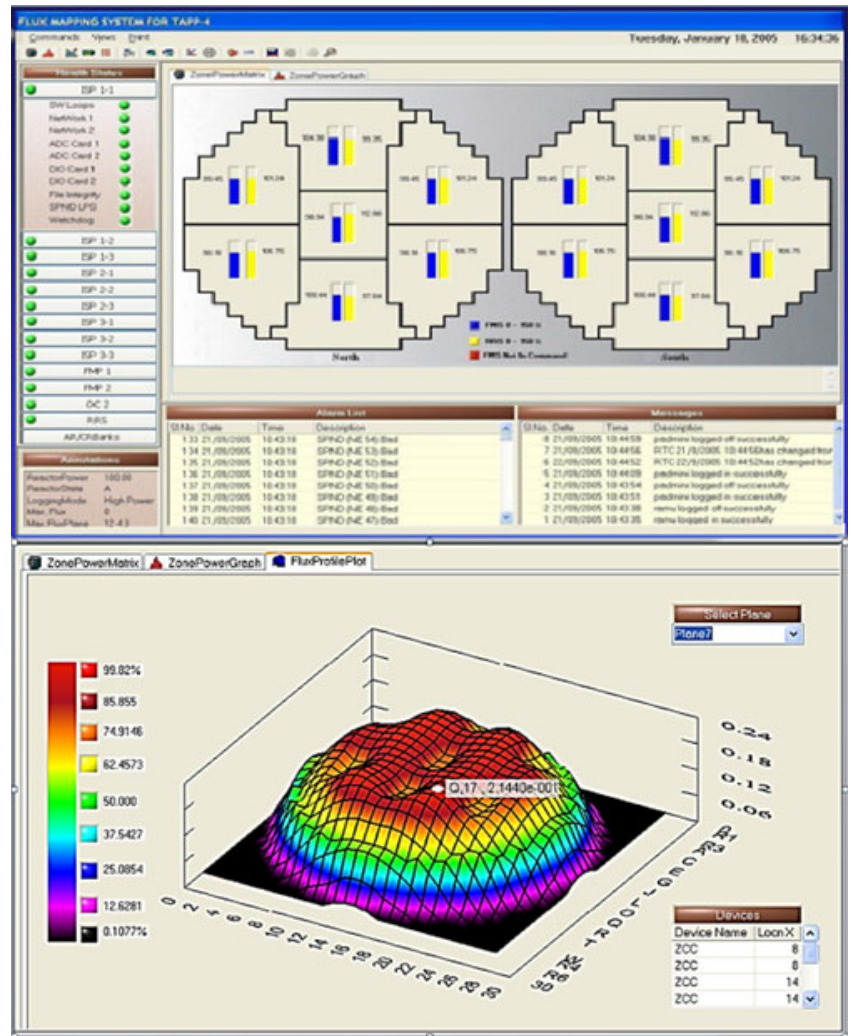

Figure 8. FMS operator console and 3D flux map.

Shut down systems of the reactors are required to act and shut down the reactor to achieve and maintain safety and is a safety class system. PHWRs have two shut down systems (SDS). The first computer-based safety system was deployed at Kakrapar for SDS-1 and was known as Programmable Digital Comparator System (PDCS). It has replaced more than hundred IAMs and is generating trips and alarms and is also providing centralised information to operator on all these signals. In later reactors, it has undergone continuous improvements with respect to technology and features. In Tarapur, a separate system of PDCS has been augmented for safety-related functionalities.

Testability of safety system is a design requirement as per AERB safety guide. Computerbased test and monitoring systems have been built and used in various plants. Systems which do on-line test and off-line tests are deployed for various systems, based on the requirement. Computer-based systems are that elaborate and complete self-diagnosis can be built into the system, which can pinpoint the faulty module and hence reduce the MTTR. Hardware is made modular and easily replaceable on-line. The computing power of computer, combined with high resolution video screens make it possible to present the data to operator in graphical formats, trends, graphs, mimics, etc. for effective operation. It is possible to record data and maintain archive for long period, for analysis and generate reports. It is also possible to filter the record and alarms based on various categorizations, during analysis. All the computations and comparisons are done in digital domain which does not have problem of drift, which is inherent in analog circuits. 
Computer-based systems can be stand-alone computers or it can be communicating to other computers or display computers. Technology know-how has improved to the extent that distributed control systems, are designed and deployed, where required. The configuration and architecture of the systems are chosen to be dual-redundant hot-standby or TMR or multi-nodal based on functionality, isolation, redundancy requirement and on consideration of geographical distribution of process. Failure Mode Effect Analysis and reliability analysis are done on the systems.

\subsection{Standardization efforts}

Industries which do not have highest level of safety concerns utilize the advances made in the automation field through the use of PLCs and Distributed Control Systems (DCS). But, use of such programmable Commercial Off-The-Shelf (COTS) systems in nuclear facilities poses qualification issues. Either the product vendor has to produce requisite quality certificates or has to agree to subject their design to the required level of scrutiny.

In-house development of programmable I\&C platform as building block facilitates a I\&C architecture where multiple platforms performing different $\mathrm{I} \& \mathrm{C}$ functions are connected over layers of networks - for data acquisition, control communication and plant information. The command and control flows down to the individual systems over dedicated communication networks unless it is safety critical, in which case it is hardwired.

Following this approach, Trombay Programmable Logic Controller-32 (TPLC-32), was developed based on 32 bit CPU. The Standalone platform can be used in isolated triplicated configuration. Hot-standby and distributed configurations were worked out based on standalone platform to meet the reliability and availability requirements of different control and protection systems. The Application Development Environment (ADE) for TPLC-32 provides for configuration of hardware, I/O, network and alarms so that the application development can be integrated in one place. Extensive checking has been implemented to detect inconsistencies among various configuration data items.

As a versatile I\&C platform, TPLC-32 is being used in the current and future requirements of I\&C systems. As part of modernization of Dhruva Reactor, four safety I\&C systems i.e., Reactor Trip Logic System, Start Up Logic system, Emergency Core Cooling Logic and Alarm Annunciation System have been successfully developed using TPLC-32 Platform and are in the stage of deployment in Dhruva.

Distributed Control System for Integrated Project for Nuclear Recycling is being developed as one of very large-scale installation using TPLC-32 based building blocks. The integrated plant is distributed in six process blocks with an I/O count of around 25000. Control system for the integrated plant is configured in a distributed architecture (figures 9 and 10) with control algorithms for each process block implemented in dual redundant TPLC-32 based programmable controllers. The I/O is distributed in multiple remote nodes connected with redundant communication link.

\subsection{Development of modern control room}

BARC is developing AHWR as a next generation nuclear power plant. A modern control room has been conceived for operation and monitoring of the plant. Design of the modern control room was aimed to reduce workload on operator, enhance situation awareness, early detection of abnormal situations and effortless handling of emergency conditions thereby improving overall safety and availability of the plant. The design has been finalized with a control room having 


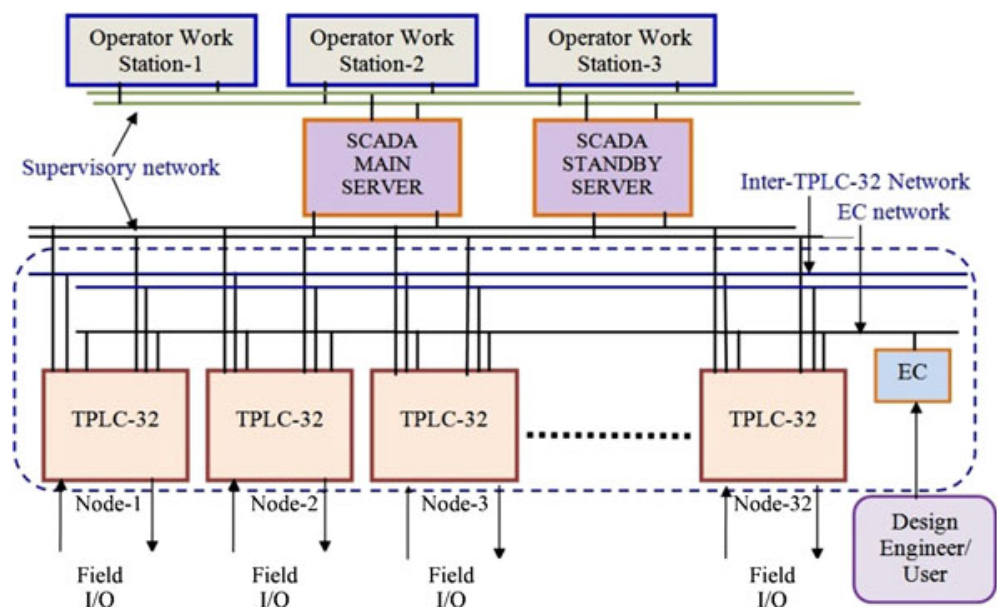

Figure 9. TPLC-32 based distributed I\&C system.

fully computerized operator consoles and shift supervisor console incorporating computerized safety parameter display system (SPDS), alarm processing and presentation and other operator support systems. A scaled down prototype of the operator console was used to evaluate the design aspects of the soft control interfaces for the operator interaction. The set-up consists of the operator console for the reactor side with display stations along with the large display panel as shown in figure 11. Various HMI schemes conceptualized and verified include, Human Machine Interface Design, Function oriented display on a large display panel.

\subsection{Radiation monitoring in nuclear power plants}

The general safety requirement for a nuclear power plant demands that nuclear power plant operation should not cause radiation hazards, which could endanger safety of workers or population

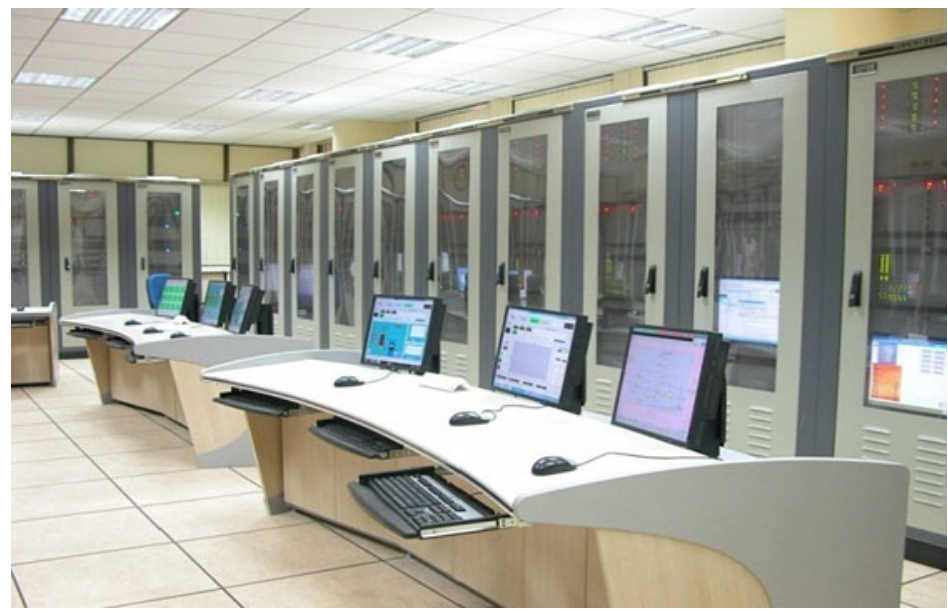

Figure 10. Prototype set-up of distributed TPLC-32 system. 


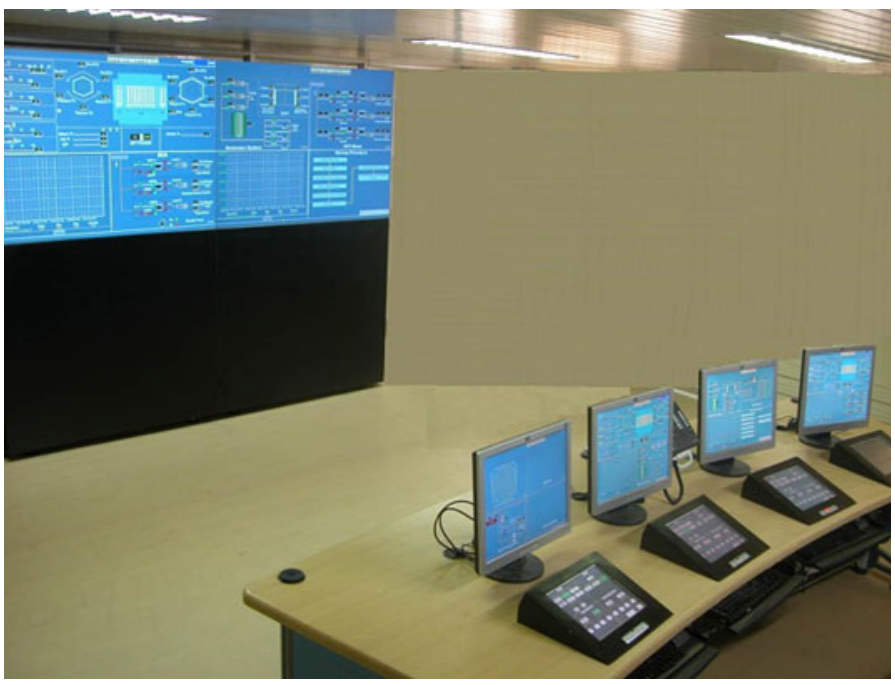

Figure 11. Prototype control room set-up.

in the vicinity or could otherwise harm the environment or property. The safety requirement is achieved by radiation and environment monitoring system continuously operating in the plant consisting of various types of radiation monitor, on line signal processing and data acquisition units and display of radiation levels and warning and emergency conditions of the plant on a central operator console.

The main function of a Radiation Monitoring System (RMS) is automatic and remote monitoring of radiation environment of the whole plant for radiation and personnel safety by control of radioactive leakages. The radiation exposures and radioactive leakages are monitored by means of a large number of various types of monitoring instruments for Gamma, alpha and beta, and neutron radiations. The operational health of the plant is continuously monitored by means on line beta gaseous activity monitors, which measure radioactive leakages in gaseous phase in the vicinity of reactor viz. reactor room, pump room and steam generator process line. The core integrity and fuel failure is monitored by continuous on-line monitoring of reactor power and coolant activity build-up. It is ensured that following measures are met during emergency conditions of plant: Measurement of dose rate in the reactor containment, determination of the concentration of radioactive substances in gas phase inside the containment, determination of radioactive leakage in the coolant.

The RMS is a plant wide networked system and consists of permanently installed (stationary) and portable radiation monitoring systems and equipment The RMS ensures the radiation monitoring in emergency and accidental conditions of the plant. For the purpose of accident monitoring and management, appropriate measuring and monitoring instrumentation is designed by which the operating personnel obtains sufficient data for event assessment and for the planning and implementation of counter measures. The measurement systems designed for accident monitoring and management maintains operability even in the event of a single failure.

\subsection{Fuel handling control systems for Indian nuclear power reactors}

For generating power on continued basis, on-power refuelling is essential in PHWRs. The refuelling of the reactor is carried out after opening the reactor pressure boundary viz. opening the 
high temperature and high pressure channel of a reactor at two ends of the coolant channel, while reactor continues to produce power. This operation is carried out by two remote controlled Fuelling Machines working in synchronism and Fuel transfer equipment, which together perform more than 1200 varied and complex robotic operations involving highly precise movements. This large number of operations must be performed in a predefined sequential order, satisfying the complex interlocks based on continuous monitoring of various signals. From the very start, it was decided that Auto-Mode operation with minimal (near zero) human intervention is preferable over Manual Mode as to nullify the consequent negative effects due to human errors. The refuelling operation is automatic, once the operator selects the channel to be refueled, the number of bundles to be loaded/unloaded and sequence desired to be executed.

Fuel handling control systems have undergone progressive technological advances over four decades. Earlier PHWRs at RAPS-1\&2 and MAPS-1\&2 are based on hardwired system design. Control system configuration, similar to the BARC test facility, were commissioned at NAPS-1 and NAPS-2 in the year 1988 and 1991, respectively and later at KAPS-1\&2. For RAPP-3, 4, $5 \& 6$ and KGS-1, 2, 3\&4 the concept of master slave was replaced by two in-house developed 8086-microcomputers. An operator interface system with operation logging in disc was provided using PC. The control system software was validated using Industrial PC-based dynamic simulator specifically developed for the purpose. This not only enabled automatic and thorough testing of the interlock and refuelling sequence logic but also the dynamic behaviour of the control system in real time as it also implements mathematical model of various $\mathrm{FH}$ components.

For the two 540 MWe PHWRs at Tarapur, the control system development utilized in-house developed 8086 microcomputers and advanced software technologies. Many innovative features in the design of mechanical assemblies of Fuelling Machine and Ram assembly needed development of complex control algorithms. Subsequently, full-fledged control system was developed and commissioned at Tarapur in the year 2004. The control system has full operability through PC-based operator interface system. FH Training Simulator (FHTS) for PHWRs.

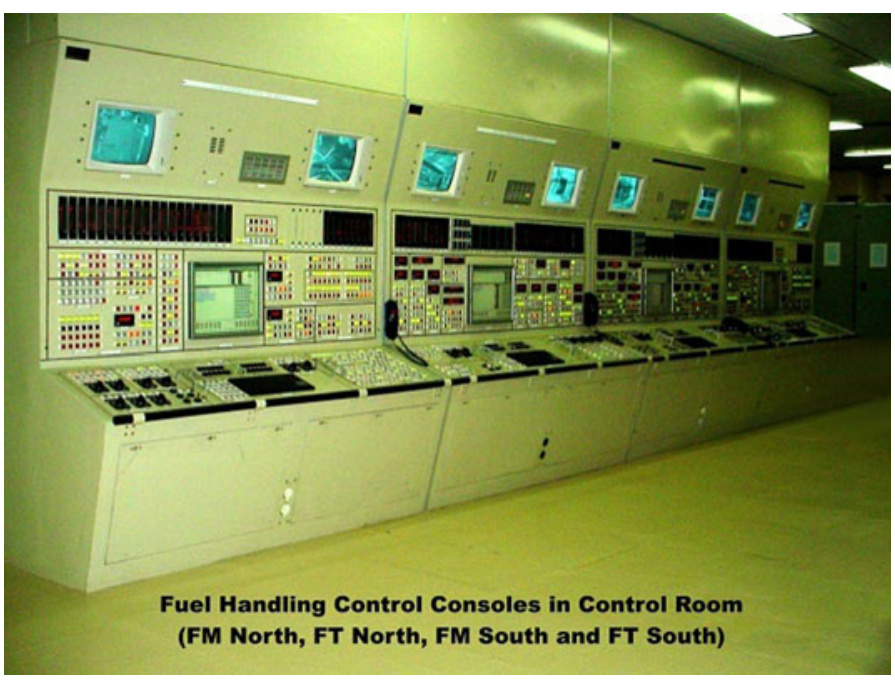

Figure 12. Fuel handling control consoles in control room. 
Though the on-power refuelling in PHWRs are carried out in Auto Mode using Computerized Control System, skilled and trained O\&M crew are necessary to overcome the off-normal and emergency situations in addition to normal refuelling operation. The first FHTS system was commissioned at Nuclear Training Center (NTC), Kaiga in 2002 for 220 MWe. The advanced version around a network of 14 PCs and two 42-inch 'plasma display panels (PDPs)' for display of 3D graphics associated with on-line and off-line mimics in real time was developed for 220 MWe and 540 MWe, and installed at NTC, Kota and NTC, Tarapur. Fuel Handling Control Consoles in control room is shown in figure 12.

For 500 MWe Prototype Fast Breeder Reactor (PFBR), shutdown refuelling is envisaged in every eight months to replace approximately 100 various sub-assemblies e.g., Fuel, Blanket and Absorber. VME based Fault tolerant Dual redundant hot standby Real Time Computers (RTC) based on 68020 processor with Switch Over Logic Circuit (SOLC) is used for controlling IFTM \& CTM of PFBR. The RTCs are placed inside RCB and communicate to PC based Operator Interface and Plant Process Computer using dual Safety Class II data highway. RTC system health (hardware as well as software) is continuously monitored by on-line diagnostics and Watchdog timer. RTC system health pulse is used to switchover to standby RTC, in case the primary RTC fails. PFBR refuelling schematic involving various machines is shown in figure 13 .

\subsection{Plant simulators for nuclear power plants}

Plant simulators play a very important role in our nuclear power programs. The computer-based simulators, which execute mathematical models of the plant, are used for: operator training and control system testing in nuclear technology.

The simulator design considerably differs depending on the type of simulator. The simulator development starts after its purpose, scope and all functional and performance requirements are

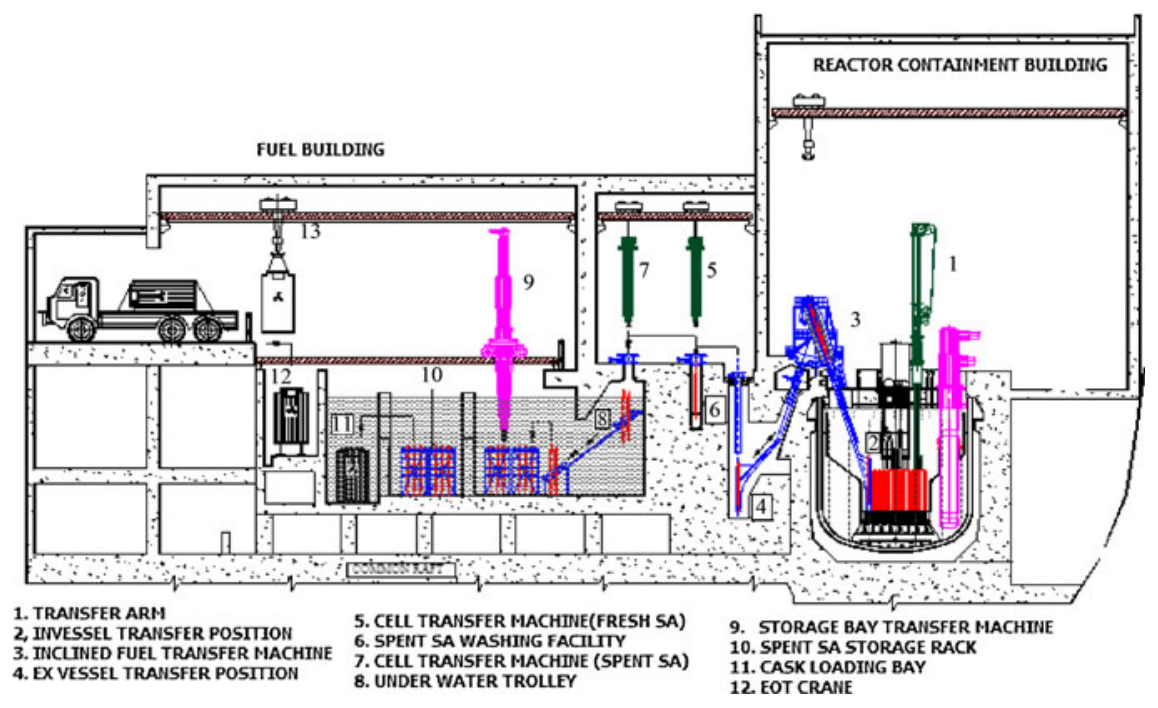

Figure 13. PFBR refuelling schematic involving various machines. 
clearly spelt out in a document. IAEA standard (IAEA TECDOC 1992) helps in preparation of the requirements document. Following are the important features that should be covered for a training simulator:

- Plant processes and control systems that are to be modelled

- Operator consoles

- Various states of the plant, from which simulation can start

- List of failures of processes, equipment and control systems that are to be simulated

- Facility for monitoring, recording, archival and retrieval of operator's actions and plant responses.

Since mathematical models are never perfect, process modelling requires considerable experience and knowledge base. It is challenging to design failures as these are numerous and care should be taken, otherwise, failure injection may violate boundary conditions of the numeric algorithm leading to instability and subsequent crash of software. The hardware architecture, with the use high speed LAN, is also very important to ensure real-time feedback to the operator.

The most strenuous part of any simulator development is its validation or testing. The simulator performance is compared against a standard model, which are built from results of experiments on a similar plant or other simulation codes that have been validated. Unlike the other types of software, the number of iterative cycles (modify-test-modify...) of simulator software is high, as each correction makes it possible to execute more scenarios and thus revealing more latent errors. A formal validation document is necessary that ensures completeness of the checks with respect to all the requirements.

In the recent years, BARC has developed NPP simulator for LWR (refer figures 14 and 15), which is a full scope simulator for training of operator. The simulator is a distributed computer system connected by dual Gigabit LAN and has replicas of the control panels.

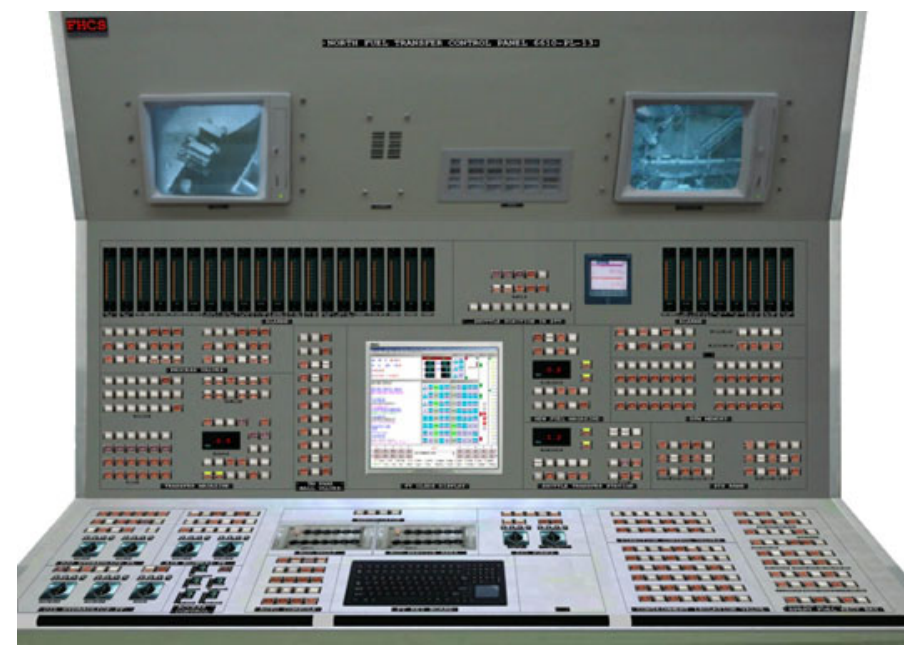

Figure 14. Fuel handling simulator. 


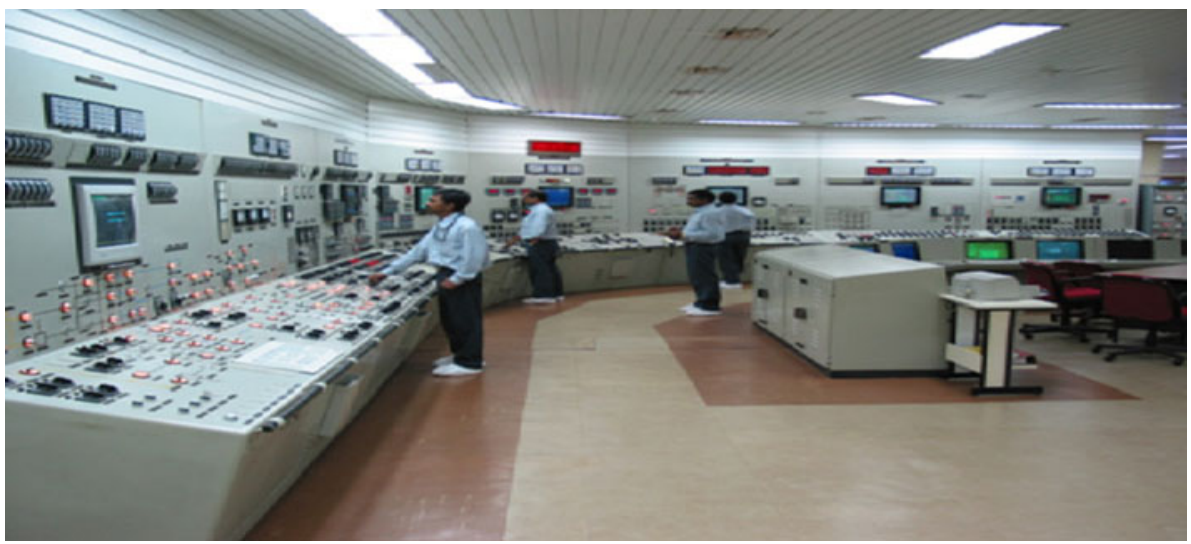

Figure 15. Full scope simulator at an NPP site.

\section{Dependability of instrumentation \& control (I\&C) software}

Nuclear Power Plants in India have been using software-based I\&C systems for more than 3 decades. But, it was only from late 1980's that software has shown its presence in safety-critical systems of Indian NPPs. Because of their criticality with respect to plant safety, development of these software packages has distinct characteristics from that of commercial software or information system software. BARC has been in the forefront in the development of these critical software packages for Indian NPPs.

It is not sufficient to ensure dependability (e.g., by thorough testing, formal verification, etc.), but it shall also be demonstrable through documentary evidence. For this, it is necessary to follow a systematic and controlled software development process. Important elements that assure dependability are: requirements analysis, standards compliance, verification \& validation, planning and documentation.

Requirements analysis is one of the most critical tasks in development of dependable software. Sometimes, the omissions in requirements specification are not detected till the operational phase, which leaves considerable risk to the plant. Use of formal or semi-formal languages has helped reduce the ambiguities and incompleteness in requirements specification. Unified Modelling Language (UML) is one of the semi-formal languages that have been used for specifying requirements.

The second element related to assuring dependability is compliance to relevant and applicable standards. AERB-D-25 (AERB/SU 2010) is the guide that has been used for safety systems of an Indian NPP. Other widely used international standards are: IEC 60880 (IEC 608802006 ) and IEC 62138 (IEC 62138 2004). The standards specify: requirements for development, V\&V, quality assurance and change management; list of documents to be produced; do's and don'ts in the design; and some important design criteria.

The third element for assuring dependability is $\mathrm{V} \& \mathrm{~V}$, which also includes testing. Verification checks if product of a software development phases meets the requirements set forward in a previous phase, while validation checks, at the end of development, if the final software product meets its requirements. Phase-wise V\&V using automated tools is being used to better efficiency and coverage. Besides, formal methods have been reported, for the most critical software, to ensure compliance of the design to the requirements. Peer-V\&V has been done which has been very successful in eliminating the software errors early in the development phase and in disseminating information about possible flaws and common mistakes in the design process. As per 
regulatory requirements in India, software of the highest safety class needs to be subjected to $\mathrm{V} \& \mathrm{~V}$ by a third party independent of the designers and users. In the area of testing, coveragebased and tool-assisted automated tests are used. Also, statistical testing and reliability growth techniques have been used to assure high dependability level for the software.

A documented software development plan at the beginning of a project is a very important element in assuring dependability. Typically, the plans consists of software project management plan (SPMP), software quality assurance plan (SQAP), software V\&V Plan (SVVP) and software configuration management plan (SCMP). These plans stream line the development, specify the activities that are performed (e.g., QA checks) and reports that are generated to provide documentary evidence (e.g., for regulatory body) that the development indeed was executed as planned.

Documentation provides evidence that an appropriate system of development and $\mathrm{V} \& \mathrm{~V}$ has been used. It also makes the products (requirements, design) amenable to review so that anomalies are resolved at the earliest. Over the last two decades size of NPP software documents have increased more than four times, indicating the importance given to the documentation.

Model-based development (MBD) is becoming popular, in which software requirements are specified in application specific language, e.g., using block control language. The development tool ensures that translation from the specification to the final code is correct; some tools use formal techniques for this purpose. MBD reduces coding errors and drastically cuts-down the development and $\mathrm{V} \& \mathrm{~V}$ effort and time. But, one requires very high confidence on the tool (as manual intervention in $\mathrm{V} \& \mathrm{~V}$ is cut-down drastically).

The tool-based verification support for computer-based systems, makes it rigorous, repeatable, and time bound. Various areas in the system development life cycle have been identified where such verification tools need to be deployed. Although commercial solutions are available in some areas for verification of software and hardware, scalability, technological obsolescence, and supply availability from foreign vendors always remain in question. This is the main motivation behind the ongoing programme in BARC to develop indigenous tools for software and hardware verification. This also includes program analysis and verification tools that have been used for verification of critical safety requirements.

During the initial phase of introduction of computer based systems (CBS), the first such system used for nuclear safety was developed entirely using assembly language. Qualification of the software used in this system required a comprehensive review. However, during that period there was no tool available to us. One of the early contributions to address this was the development of static analysis tool (ALPS) for analysing control and data flows in assembly language program. This tool was used quite extensively in the review of the software performing safety functions in Programmable Digital Comparator System (PDCS). Subsequently, we have developed static analysis tools (CLAS) for other Language like 'C' programming Language. Expertise was also developed in using commercial tools like LDRA Testbed and Polyspace for static program analysis. During the regulatory clearance of I\&C systems, this expertise was very useful in qualifying the systems as per the established process in AERB.

There has been a strong emphasis on Formal Verification of Software with an aim to verify software programs against High Level Requirements (HLR). This had led to the development of ACE (Assertion Checking Environment) toolsets based on Hoare logic which use Theorem Provers like PVS (Prototype Verification System) and SteP (Stanford Temporal Provers) at the backend. The tool has been used for verification of implementation of trip logics in Nuclear Power Reactors.

Many I\&C systems are now increasingly using FPGA devices, which allow configurability by the hardware designer. The configurability is enabled by the capability of 'field programming' such hardware devices using Hardware Description Languages (HDL) like VHDL. Since the 
functional bugs in such hardware subsystems used in I\&C of safety critical systems have disastrous consequences, it is very much required that verification techniques of equal rigour as of software should also be applied to design of such hardware components. Recently, we have completed the development of a hardware verification tool VBMC (VHDL Bounded Model Checker) based on the principle of bounded model checking. The tool has been used for verification of in-house designed logics implemented in FPGA.

One of the strong points in this activity is the stress on developing algorithmic solutions for formal verification with academic rigour. A framework has been developed for active collaboration with academic community in this programme and has supported extramural research in these areas. One of the notable contributions in this area has been the activity being supported in Centre of Formal Design and Verification of Software (CFDVS) at IIT Bombay.

NPPs extensively use computer-based systems in safety-critical applications also, in which a security compromise can potentially cause failures and extensive damage to the plant and environment. The system security or, in this context, I\&C security deals with protecting I\&C system against unauthorized modification of its resources or disruption of its services.

Three components of I\&C security are: security controls, defense-in-depth and security lifecycle. Security control includes plans, policies, procedures, and other technical, operational and management controls during development, deployment and operation of the system. The security control is put in place after understanding the vulnerabilities of the target system-which is to be secured- and its operating environment. Impact due to various security failures are analysed so as to put in place adequate counter-measures as part of the security controls. The security controls as applied to information systems and $\mathrm{I} \& \mathrm{C}$ systems are fundamentally different as traditional security controls such as periodic updates cannot be applied to I\&C systems due to its environment characteristics and limited resources.

Defense-in-depth philosophy implies that the most critical systems are kept at the core, which is protected by many layers of barriers, with each barrier characterized by a more stringent access control and information flow control. In a plant, this is typically implemented by creation of several zones in which I\&C systems are placed depending on their criticality to the plant safety. Information flow and access between the zones are controlled through firewalls in what is called de-militarized areas. The most critical systems would not have any network connections to any external systems or networks to eliminate the most common vulnerabilities.

Security life cycle has been a very important component to ensure I\&C security. Normally, software/system development cycle pass through a number of phases and verification is executed in between the phases to ensure that all functional and performance requirements of the software/system are correctly implemented. However, in case of security, it is a never-ending process till the system is de-commissioned. During the development, the development life cycle process should ensure that the verified and validated software/system only - without any malicious modification-is deployed. As new vulnerabilities in computer-based systems are thrown up each day, during operation, periodic review should be conducted to study relevance of newly detected vulnerabilities, and to assess efficacy of existing security control to thwart any cyberattacks on the I\&C systems. If required, security controls should be appropriately updated to make it more effective. Review of security controls will also be required if any modification is done in the software/hardware and there could be new vulnerabilities associated with the modified parts.

Access and control of computer system and its resources are governed by the operating system (OS). The necessity of operating system security to overall system security is undeniable; the underlying operating system is responsible for protecting application-space mechanisms against tampering, bypassing, and spoofing attacks. Hence, OS is a very logical place to enforce and support security and it is essential to make the OS as hardened as possible to protect the 


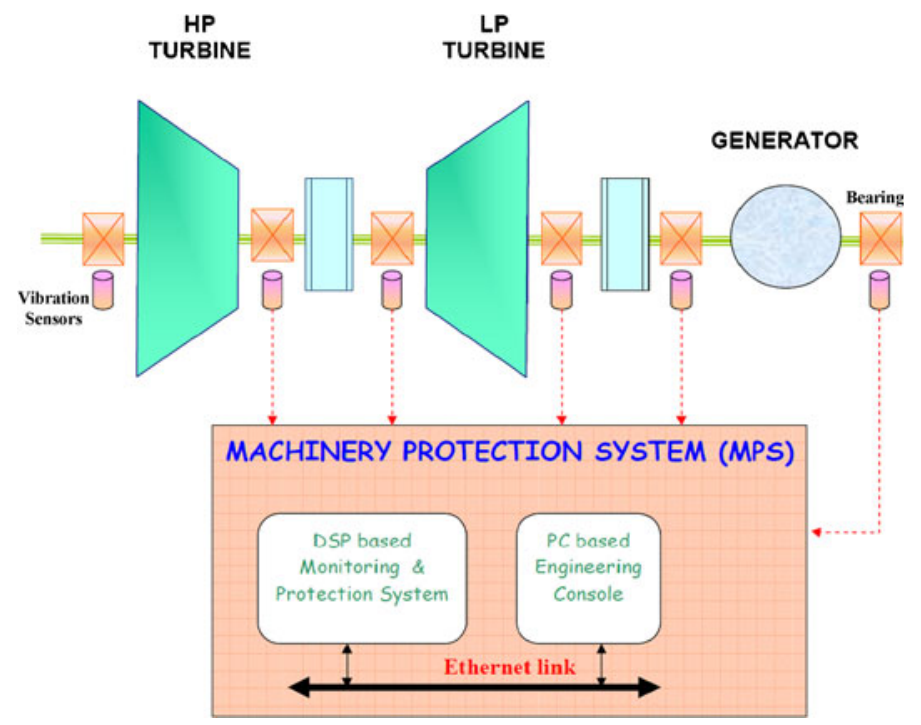

Figure 16. Functional diagram of machinery protection system.

system from various cyber-attacks. Based on this assessment, BARC is striving to make our own operating system that will be hardened to minimize and, if possible, eliminate the vulnerabilities.

\section{Diagnostic tools and equipments}

BARC has ushered in a number of technologies which are first of their kind in the country. As a part of the effort towards overall energy security of the country diagnostic tools and nondestructive testing and evaluation systems have been built using inspections technologies such as vibration, current signature, ultra sonic (UT), magnetic flux leakage (MFL), acoustic emission and eddy current.

Health of large rotating machinery gets reflected in the vibration signals and monitoring of these signals can provide quick warning against excessive vibration so that preventive action can

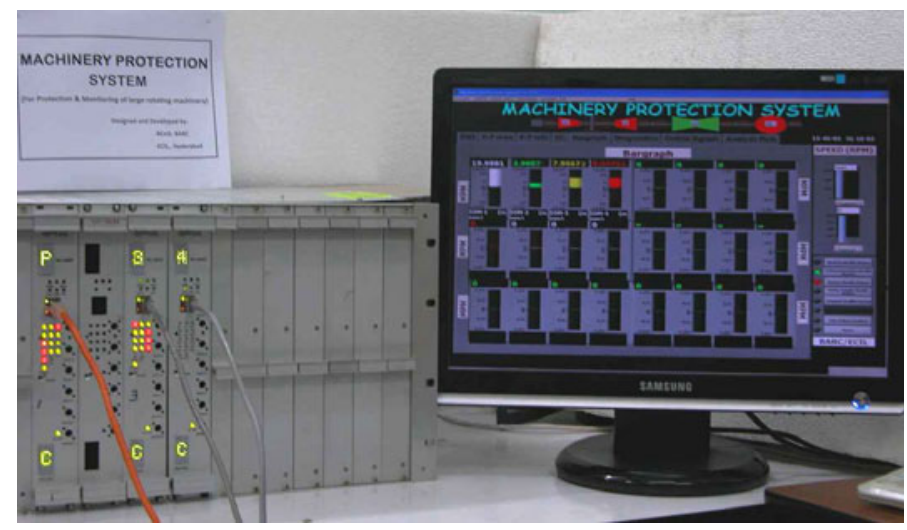

Figure 17. Machinery protection system. 
be taken in time and catastrophic failures can be avoided. A vibration monitoring and protection system thus plays an important role in ensuring safety and economics of the plant (refer figures 16 and 17). To monitor the vibration of machinery like Turbo-Generator sets and large pumps in nuclear power plants and to provide protection against failures due to excessive vibration, a

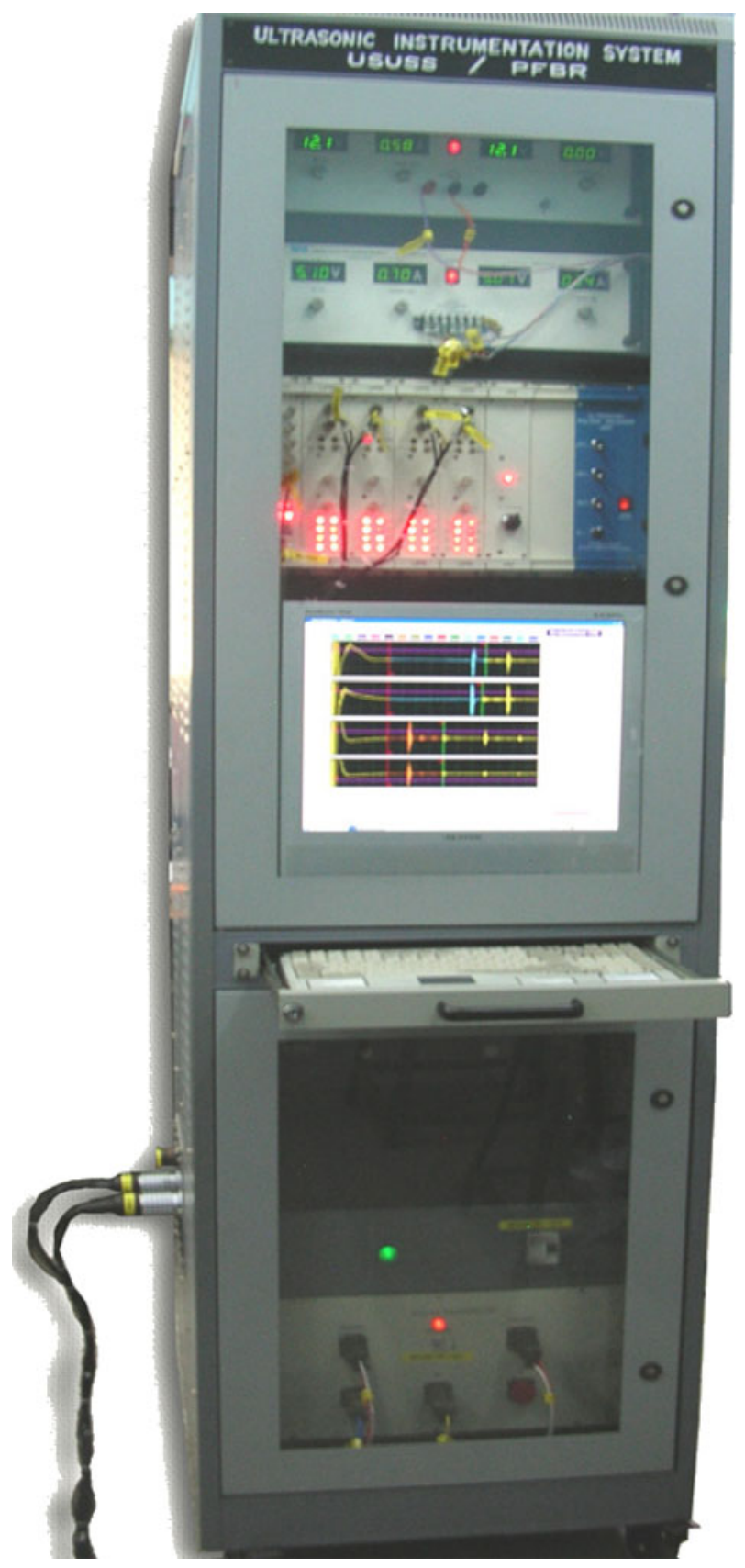

Figure 18. 8-Ch ultrasonic imaging system. 


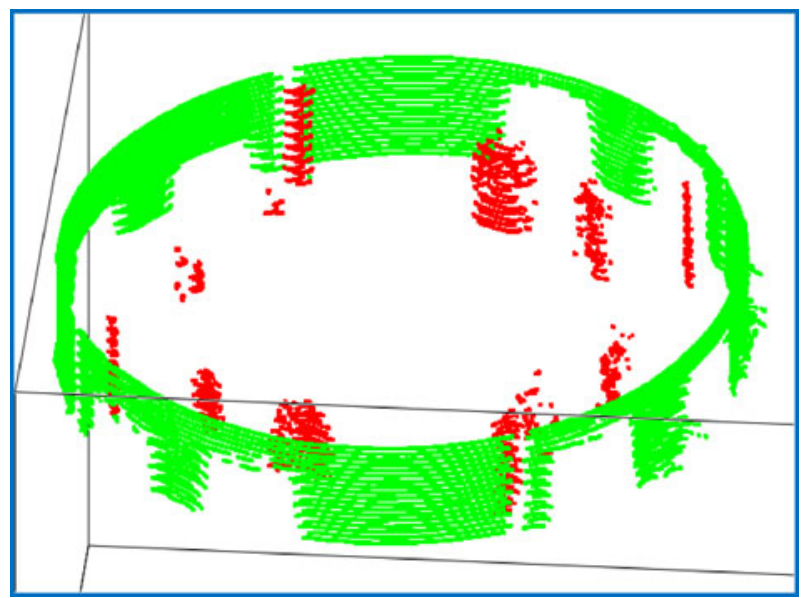

Figure 19. 3D Ultrasonic image of in-sodium FSAs using SVT.

Machinery Protection System (MPS) has been designed at BARC. The DSP based Machinery Protection System is capable of processing up to 24 vibration and speed signals and these are continuously monitored to check for any deviation beyond the set alarm and trip limits. The system can be interfaced with different types of sensors for monitoring bearing and shaft vibration, shaft position and eccentricity, absolute and differential casing expansion and tachometer speed signals. Its Signal Processing functions include multi-rate FIR Digital filters, harmonic content analysis and determining RMS, Mean and Peak-to-Peak values of signals. It generates digital alarm output on alarm conditions and provides trip signal for critical alarms by relay actuation. The system has passed acceptance tests and integration tests at ECIL and is being deployed in working power plants for field trials. The system will be taken up for manufacture by ECIL and will be available to users like nuclear and thermal power plants with long term maintenance support.

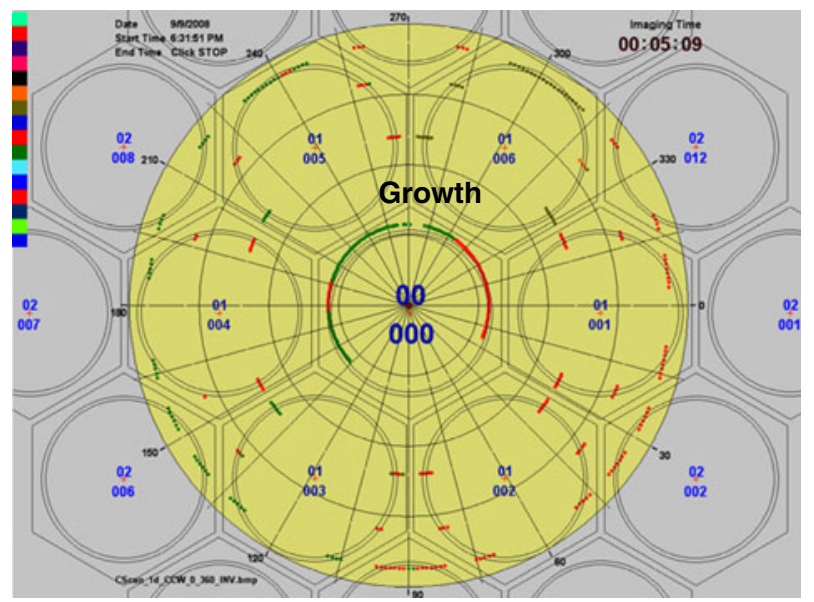

Figure 20. Detection of growth of in-sodium fuel sub-assembly. 
PFBR is a sodium-cooled fast breeder nuclear reactor which has sodium-submerged 181 Fuel Sub-Assemblies (FSAs) in the core of the reactor. During normal operation of the reactor, the temperature of liquid sodium is more than $550^{\circ} \mathrm{C}$. Due to high temperature, prolonged irradiation and flow of liquid sodium, there is a possibility of growth and protrusion of FSAs. Ultrasound is the way available for visualization inside the optically opaque liquid sodium. An automated Under Sodium Ultrasonic Scanner (USUSS) has been designed and developed (figures 18, 19 and 20) for detection of growth and protrusion of FSAs during shut-down state of PFBR when the temperature of liquid sodium is $180^{\circ} \mathrm{C}$. Ultrasonic Pulse-Echo mode and C-Scan imaging technique are employed to detect growth and protrusion of FSAs. To scan the core of PFBR, sodium-compatible ultrasonic transducers of $5 \mathrm{MHz}$ are employed for downward viewing operation for detection of growth in FSAs which are located underneath the scanner, whereas $1 \mathrm{MHz}$ transducers are utilized to scan in lateral i.e., sideways direction for detection of protrusion of FSAs as well as getting positional status of Control and Safely Rod and Diverse Safety Rod drive mechanisms. The scanning is carried out before fuel handling operation for safe movement of Large and Small Rotatable Plugs of PFBR. The 8-Channel Ultrasonic Imaging System consists of an Industrial grade PC, digitizer, 8-Channel ultrasonic tone-burst pulser-receiver and Linux-QT based imaging software.

\section{Silicon detectors and ASIC}

Silicon detector development has been carried out at BARC to indigenously develop a wide range of detectors having different specifications in terms of detector geometry, area, segmentation and energy resolution. These detectors include large area silicon strip detectors and micro strip detectors, silicon pad detectors, high energy resolution detectors for alpha and charged particle spectroscopy, single and multi-element silicon PIN photodiodes, radiation sensitive MOSFETs and silicon PIN diodes for dosimeters. The strip detectors are being used for $\pi^{\circ} / \gamma$ rejection for the search of Higgs bosons. Novel detectors such as integrated $\Delta \mathrm{E}-\mathrm{E}$ silicon detectors which integrate a very thin detector $(10-25 \mu \mathrm{m})$ and thick detectors $(300 \mu \mathrm{m})$ in one chip have been realized for identification of charged particles as well as measurement of their energy. Development of new types of detectors such as silicon photomultipliers, double-sided strip detectors for 2D position sensing/imaging and detectors for security applications (X-ray baggage scanning and cargo scanning) is in progress.

The ASIC developments can be categorized as: Detector Signal Processing and Nuclear instrumentation ASICs which are: multichannel low noise charge amplifier and pulse shapers readout electronics catering to different detectors. Subsequent to signal processing are nuclear instrumentation Chip sets for peak detection, Wilkinson ADC and 128 cells Analog Memory. The societal application of ASIC development activity in medical instrumentation is the low power ECG signal processing ASIC. A large number of HMCs were designed and developed for various nuclear instrumentations needs and these HMCs have undergone mass manufacturing.

To augment the micro-electronics activity in a comprehensive and focused manner, a facility called CMEMS has been set-up to cater to semiconductor process and device development, IC design, characterization and spice modelling, wafer level IC testing along with packaging equipment's like flip chip bonder and wire bonder. This allows end-to-end foundry-less process to chip/device development. This focused activity has resulted in development of number of ASICs

and HMCs for various high energy physics experiments in the country and portable radiation monitoring instruments. 


\section{Conclusion}

Achievements in $\mathrm{I} \& \mathrm{C}$ activities in the areas of detector development, nuclear instrumentation, and monitoring and control electronics have taken the country to a position of strength in global nuclear map. Very high level of safety and reliability in plant operation meeting stringent regulatory requirements is of paramount importance and the same is achieved by extensive V\&V activities and employing inspection systems. The paper gives an overview of the developments undertaken at BARC in the field of electronics to cater to the basic requirements of Indian NPPs. It is largely motivated by the need for self-reliance in the areas of strategic developments that ensured sustenance of the indigenous nuclear program.

\section{Acknowledgement}

I thank my colleagues Ms Padmini S, Scientific Officer (H) of ED, BARC and Mr S Mukhopadhyay, Scientific Officer $(\mathrm{H})$ of CnID, BARC for their support in organizing and compiling this paper.

\section{References}

Dubridge, R A 1967 Campbell Theorem, system concepts and results, IEEE Transactions on Nuclear Science, February

IAEA TECDOC 6851992 Simulators for training Nuclear Power Plant Personnel, IAEA, Vienna

AERB/SG/D-25, January 2010 Computer Based Systems of Pressurised Heavy Water Reactor, AERB Safety Guide

IEC 60880 2006-05, Nuclear power plants-Instrumentation and control systems important to safety Software aspects for computer-based systems performing category A functions

IEC 621382004 Nuclear power plants-Instrumentation and control systems important to safety Software aspects for computer-based systems performing category B and C functions 